\title{
Necessary Conditions for Fredholmness of Partial Differential Operators of Irregular Singular Type
}

\author{
By \\ Masatake MiYaKe* and Masafumi Yoshino**
}

\section{Introduction}

The convergence of formal power series solutions of ordinary differential equations are extensively studied by many authors in connection with irregularities. (cf. [6] and [10].) In case of partial differential equations of regular singular type, several sufficient conditions are known. (cf. [4] and [1].) In the preceeding paper [9], we gave sufficient conditions for the Fredholmness of partial differential operators of irregular singular type of two independent variables in analytic and Gevrey spaces. Then we deduced the convergence of formal power series solutions from the Fredholmness of the operators. These conditions are expressed in terms of Toeplitz symbols, and they are equivalent to a Riemann-Hilbert factorization condition. (cf. [3] and (2.5), (2.6), (2.7) which follow.) In this paper, we shall show the necessity of these sufficient conditions. More precisely, we will prove that the Riemann-Hilbert factorization conditions (2.6) and (2.7) are necessary and sufficient for the partial differential operators of irregular singular type to be Fredholm operators on certain analytic and Gevrey spaces.

The proof of our theorem is based on the analysis of the main (principal) part of irregular singular type operators via Toeplitz operators on the torus $\mathbb{T}^{2}:=\mathbf{R}^{2} / 2 \pi \mathbb{Z}^{2}$. In fact, we will show that the essential parts of these operators in studying Fredholm properties are precisely Toeplitz operators. This

Communicated by T. Kawai, December 18, 1995.

1991 Mathematics Subject Classifications : primary 35C10 secondary 45E10, 35 Q15.

*Department of Polymathematics, Nagoya University, Nagoya 464-01, Japan.

${ }^{* *}$ Faculty of Economics, Chuo University, 742-1, Higashinakano, Hachioji, Tokyo. Japan.

Key words and phrases. necessity for Fredholm property, irregular singular PDE, Toeplitz operators, Riemann-Hilbert factorization.

The second author is partly supported by Grant-in-Aid for Scientific Research (No. 07640250), Ministry of Education. Science and Culture, Japan and by Chuo University Special Research Fund. (1995). 
enables us to give necessary and sufficient conditions for Fredholmness in terms of Toeplitz symbols.

\section{\$2. Statement of the Results}

Let $\mathbb{N}$ be the set of nonnegative integers and $\mathbb{C}$ be the set of complex numbers. Let $\mathbb{C}[[x]]$ be the set of all formal power series

$$
\mathbb{C}[[x]]:=\left\{u(x) ; u(x)=\sum_{\eta \in \mathbb{N}^{2}} u_{\eta} \frac{x^{\eta}}{\eta !}, \quad u_{\eta} \in \mathbb{C}\right\}
$$

Let $w_{j}>0(j=1,2)$ and $s>0$, and let us set $w=\left(w_{1}, w_{2}\right)$. We denote by $\mathscr{O}$ $\left(\left\{\left|x_{1}\right|<w_{1}\right\} \times\left\{\left|x_{2}\right|<w_{2}\right\}\right)$ the set of holomorphic functions in a domain $\left\{\left|x_{1}\right|<w_{1}\right\} \times$ $\left\{\left|x_{2}\right|<w_{2}\right\} \subset \mathbb{C}^{2}$. We define the space $G_{w}^{s}(\mu)(\mu \in \mathbb{R})$ by

$$
G_{w}^{s}(\mu):=\left\{u=\sum_{\eta} u_{\eta} \frac{x^{\eta}}{\eta !} ;\|u\|:=\left(\sum_{\eta}\left(\left|u_{\eta}\right| \frac{w^{\eta}}{(|\eta|-\mu / s) !^{s}}\right)^{2}\right)^{1 / 2}<\infty\right\},
$$

where the factorial is understood as the gamma function, $r !:=\Gamma(r+1)$ for $r \geq$ 0 and where we set $(|\eta|-\mu / s) !=1$ if $|\eta|-\mu / s \leq 0$. The space $G_{w}^{s}(\mu)$ is a Hilbert space with the norm $\|\cdot\|$. We note that if $s=1$, the space $G_{w}^{s}(\mu)$ is a Hardy space.

We denote by $\partial_{1}^{-1}$ the integration with respect to $x_{1}, \partial_{1}^{-1} u(x)=\int_{0}^{x_{1}} u\left(y_{1}\right.$, $\left.x_{2}\right) d y_{1}$. The operator $\partial_{2}^{-1}$ is defined similarly. For $\beta=\left(\beta_{1}, \beta_{2}\right) \in \mathbb{Z}^{2}$, we set $\partial^{\beta}=\partial_{1}^{\beta_{1}} \partial_{2}^{\beta_{2}}$, where if $\beta_{j}<0$ we understand that $\partial_{j}^{\beta_{s}}=\left(\partial_{j}^{-1}\right)^{-\beta_{3}}$.

Let $P \equiv P(x, \partial)$ be an integro-differential operator of finite order with holomorphic coefficients in a neighborhood of the origin of $\mathbb{C}^{2}$,

$$
P(x, \partial)=\sum_{\beta \in \mathbb{Z}^{2}} \partial^{\beta} a_{\beta}(x)
$$

where $a_{\beta}(x)$ 's are analytic functions of $x$ in some neighborhood of the origin and the summation with respect to $\beta$ is a finite sum.

By substituting the Taylor expansion of $a_{\beta}(x), a_{\beta}(x)=\sum_{\gamma} a_{\gamma \beta} x^{\gamma}$ in (2.2), we have

$$
P(x, \partial)=\sum_{\gamma \in \mathbb{N}^{2}, \beta \in \mathbb{Z}^{2}} a_{r \beta} \partial^{\beta} x^{\gamma}
$$


For $\partial^{\beta} x^{\gamma}$. we define the $s^{-G e v r e y ~ o r d e r ~} \operatorname{ord}_{s} \partial^{\beta} x^{\gamma}$ of $\partial^{\beta} x^{\gamma} \cdot$ by

$$
\operatorname{ord}_{s} \partial^{\beta} x^{\gamma}:=|\beta|+(1-s)(|\gamma|-|\beta|)
$$

Then the $s$-Gevrey order of $P$ in (2.3) is defined by

$$
\operatorname{ord}_{s} P:=\sup _{\gamma, \beta}\left\{|\beta|+(1-s)(|\gamma|-|\beta|) ; a_{\gamma \beta} \neq 0\right\} \text {. }
$$

Here and in what follows we always assume

$$
\text { the } s \text { - Gevrey order of } P(x, \partial) \text { is finite. }
$$

This implies that $P$ is of polynomial coefficients in case $s<1$.

We easily see that

$$
P(x, \partial): G_{w}^{s}(\mu+m) \rightarrow G_{w}^{s}(\mu), \quad m=\operatorname{ord}_{s} P
$$

is a bounded mapping for every $s>0$ and every $\mu \geq 0$ if $w$ is sufficiently small because $P(x, \partial)$ is an analytic partial differential operator. (cf. Lemma 3.2 which follows). We assume

(A.2) If $s=1 a_{\beta}(x)$ is a polynomial in $x$ for every $\beta$ in (2.2) such that $|\beta|=$ $\operatorname{ord}_{1} P$.

We define the torus $\mathbb{T}^{2}$ by $\mathbb{T}^{2}=\left\{\left(z_{1}, z_{2}\right) ; z_{j}=e^{\imath \theta}, 0 \leq \theta, \leq 2 \pi, j=1,2\right\}$. Then we define the Toeplitz symbol associated with $P(x, \partial)$ by

$$
L(z ; \xi):=\sum_{|\beta|+(1-s)(|\alpha|-|\beta|)=\text { ords } P} a_{\alpha \beta} z^{\alpha-\beta} w^{\alpha-\beta} \xi^{\alpha}, \quad \xi \in \mathbb{R}^{2}, z \in \mathbb{T}^{2}
$$

Theorem 2.1. Assume that (A.1) and (A.2) are satisfied. Let $s>0$ and $\mu \geq 0$ and $w$ be sufficiently small as above, and let $m$ be the $s$-Gevery order of $P$. Then $P(x, \partial)$ is a Fredholm operator on $G_{w}^{s}(\mu+m)$ into $G_{w}^{s}(\mu)$ if and only if the following conditions are satisfied.

$$
\begin{gathered}
L(z, \xi) \neq 0 \quad \forall\left(z_{1}, z_{2}\right) \in \mathbb{T}^{2}, \quad \forall \xi \in \mathbb{R}^{2}, \quad|\xi|=1, \xi \geq 0 . \\
\operatorname{ind}_{1} L=\operatorname{ind}_{2} L=0 .
\end{gathered}
$$

Here $\operatorname{ind}_{1} L\left(\right.$ resp. $\left.\operatorname{ind}_{2} L\right)$ is defined by 


$$
\operatorname{ind}_{1} L=\frac{1}{2 \pi i} \oint_{\left|\zeta_{1}\right|=1} d \log L\left(\zeta_{1}, z_{2}, \xi\right)
$$

Remarks. (a) We note that the right-hand side of (2.8) is an integer-valued continuous function of $z_{2}$ and $\xi$. Because the sets $\left\{z_{2} \in \mathbb{C} ;\left|z_{2}\right|=1\right\}$ and $\left\{\xi \in \mathbb{R}^{2}\right.$. $|\xi|=1\}$ are connected, the integral (2.8) is constant. Hence the right-hand side is independent of $z_{2}$ and $\xi$. We write this quantity by $\operatorname{ind}_{1} L$. We similarly define ind ${ }_{2} L$. We note that the conditions (2.6) and (2.7) are independent of $\mu \geq 0$.

(b) In [9] it was proved that if the conditions (2.6) and (2.7) are satisfied then the operator $P(x, \partial): G_{w}^{s}(\mu+m) \rightarrow G_{w}^{s}(\mu)$ is a Fredholm operator of index zero for every $s>0$ and $\mu \geq 0$. (cf. Theorem 4.3 of [9].) Hence, the novelity of the above theorem lies in the necessity of (2.6) and (2.7).

\section{\$3. Preliminary Lemmas}

Let $X_{j}(j=1,2)$ be a positive number and set $X=\left(X_{1}, X_{2}\right)$. We denote by $\mathscr{O}(|x| \leq X)$ the set of holomorphic functions on $\left\{x \in \mathbb{C}^{2} ;\left|x_{j}\right|<X, j=1,2\right\}$ and continuous on its closure. For $a(x) \in \mathscr{O}(|x| \leq X)$, we put $\|a\|_{X}:=\max |x| \leq X,, \mid a$ $(x) \mid$. Then we have

Lemma 3.1. Let $s \geq 1$. Assume that $a(x) \in \mathfrak{O}(|x| \leq \rho w) \quad(\rho>1)$. Then for any $U^{\top}(x) \in G_{w}^{s}(\mu)$, we have $a(x) U(x) \in G_{w}^{s}(\mu)$ and there exists a constant $C$ depending only on $\mu$ such that

$$
\|a U\| \leq C\left(\frac{\rho}{\rho-1}\right)^{2}\|a\|_{o w}\|U\|
$$

Proof. We put $a(x)=\sum A_{\gamma} x^{\gamma} / \gamma ! \in \mathfrak{O}(|x| \leq \rho w)$. Then by Cauchy's integ. ral formula, we have $\left|A_{r}\right| \leq\|a\|_{w o} \gamma ! /(\rho w)^{r}\left(\gamma \in \mathbb{N}^{2}\right)$. We put $a(x) U(x)=\sum$ $V_{\beta} x^{\beta} / \beta$ !. Then we have

$$
V_{\beta}=\sum_{0 \leq r \leq \beta} A_{\gamma} U_{\beta-\gamma} \frac{\beta !}{(\beta-\gamma) ! \gamma !} .
$$

Hence we have, for $C_{1}>0$ 


$$
\begin{gathered}
\sum_{\beta}\left(\left|V_{\beta}\right| \frac{w^{\beta}}{(|\beta|-\mu / s) !^{s}}\right)^{2} \leq\|a\|_{\rho w}^{2} \sum_{\beta}\left(\sum_{0 \leq r \leq \beta}\left|U_{\beta-r}\right| \frac{1}{(\rho w)^{r}} \frac{\beta !}{(\beta-\gamma) !} \frac{w^{\beta}}{(|\beta|-\mu / s) !^{s}}\right)^{2} \\
\quad \leq C_{1}\|a\|_{\rho w}^{2} \sum_{\beta}\left(\sum_{0 \leq r \leq \beta}\left|U_{\beta-r}\right| \frac{1}{\rho^{|r|}} \frac{w^{\beta-\gamma}}{(|\beta|-\mu / s-|\gamma|) !^{s}}\right)^{2} \\
\leq C_{1}\|a\|_{\rho w}^{2} \sum_{\beta}\left(\sum_{\gamma} \frac{1}{\rho^{|r|}}\right)\left(\sum_{r} \frac{1}{\rho^{|\gamma|}}\left(\left|U_{\beta-\gamma}\right| \frac{w^{\beta-\gamma}}{(|\beta|-\mu / s-|\gamma|) !^{s}}\right)^{2}\right) \\
\leq C_{1}\left(\frac{\rho}{\rho-1}\right)^{2}\|a\|_{\rho w}^{2} \sum_{\gamma}-\frac{1}{\rho^{|r|}} \sum_{\beta \geq \gamma}\left(\left|U_{\beta-\gamma}\right| \frac{w^{\beta-\gamma}}{(|\beta-\gamma|-\mu / s) !^{s}}\right)^{2} \\
\leq C_{1}\left(\frac{\rho}{\rho-1}\right)^{4}\|a\|_{\rho w}^{2}\|U\|^{2} .
\end{gathered}
$$

Lemma 3.2. Lel $\mu=|\beta|+(1-s)(|\alpha|-|\beta|)$ be the $s^{-G e v r e y ~ o r d e r ~ o f ~} \partial^{\beta} x^{\alpha}$. Then the map $\partial^{\beta} x^{\alpha}: G_{w}^{s}(\mu) \rightarrow G_{w}^{s}(0)$ is continuous. Moreover, for every $\varepsilon>0$ the map $\partial^{\beta} x^{\alpha}: G_{w}^{s}(\mu+\varepsilon) \rightarrow G_{w}^{s}(0)$ is a compact operator.

Proof. For the sake of simplicity we omit the suffices of $G_{w}^{s}(\mu)$ and write it by $G(\mu)$. We first show that for every $\kappa<\mu$ the injection $\iota: G(\mu) \rightarrow G(\kappa)$ is compact. Let $B \subset G(\mu)$ be a bounded set in $G(\mu)$. If we write $u=\sum_{\eta} u_{\eta}$ $x^{\eta} / \eta ! \in B$, then for each fixed $\eta$ the set $\left\{u_{\eta} ; u \in B\right\}$ is bounded. Hence, by the diagonal argument, we can choose a sequence $\left\{u^{(k)}\right\} \subset B, u^{(k)}(x)=\sum_{\eta} u_{\eta}^{(k)}$ $x^{\eta} / \eta$ ! such that for each $\eta, u_{n}^{(k)} \rightarrow u_{\eta}$ when $k \rightarrow \infty$.

Moreover we have that

$$
\begin{aligned}
\sum_{|\eta| \geq N}\left(\left|u_{\eta}^{(k)}\right| \frac{w^{\eta}}{\left.(|\eta|-\kappa / s) !^{s}\right)}\right)^{2} & \leq \max _{|\eta| \geq N} \frac{(|\eta|-\mu / s) !^{2 s}}{(|\eta|-\kappa / s) !^{2 s}} \sum_{|\eta| \geq N}\left(\left|u_{\eta}^{(k)}\right| \frac{w^{\eta}}{(|\eta|-\mu / s) !^{s}}\right)^{2} \\
& \leq K \max _{|\eta| \geq N} \frac{(|\eta|-\mu / s) !^{2 s}}{(|\eta|-\kappa / s) !^{2 s}} \rightarrow 0(N \rightarrow \infty),
\end{aligned}
$$

where $K>0$ depends only on $B$. This proves that the sequence $\left\{u^{(k)}\right\}$ converges in $G(\kappa)$.

In order to complete the proof it is sufficient to show that the map $x^{\alpha} \partial^{\beta}$ : $G(\mu) \rightarrow G(0)$ is continuous because the commutator $\left[x^{\alpha}, \partial^{\beta}\right]:=x^{\alpha} \partial^{\beta}-\partial^{\beta} x^{\alpha}:$ $G(\mu) \rightarrow G(0)$ is compact. Indeed, we have $\operatorname{ord}_{s}\left[x^{\alpha}, \partial^{\beta}\right]<\mu$. By simple calculations we have

$$
x^{\alpha} \partial^{\beta} \sum_{\eta} u_{\eta} \frac{x^{\eta}}{\eta !}=\sum u_{\eta} \frac{x^{\eta+\alpha-\beta}}{(\eta-\beta) !}=\sum u_{\eta+\beta-\alpha} \frac{x^{\eta}}{(\eta-\alpha) !} .
$$


Hence we have

$$
\sum_{\eta}\left(\left|u_{\eta+\beta-\alpha}\right| \frac{w^{\eta}}{(|\eta|) ! s} \frac{\eta !}{(\eta-\alpha) !}\right)^{2}=\sum_{\eta}\left(\left|u_{\eta}\right| w^{\eta-\beta+\alpha} \frac{1}{(|\eta|-|\beta|+|\alpha|) ! s} \frac{(\eta-\beta+\alpha) !}{(\eta-\beta) !}\right)^{2} .
$$

If $\eta$ is sufficiently large the term $(\eta-\beta+\alpha) ! /(\eta-\beta) !$ can be estimated from the above by constant times $|\eta|^{|\alpha|}$. Therefore we have

$$
\frac{(|\eta|-\mu / s) !^{s}}{(|\eta|-|\beta|+|\alpha|) !^{s}} \frac{(\eta-\beta+\alpha) !}{(\eta-\beta) !} \leq C|\eta|^{s(|\beta|-|\alpha|)-\mu}|\eta|^{|\alpha|}=C|\eta|^{s(|\beta|-|\alpha|)+|\alpha|-\mu}
$$

for some constant $C$ independent of $\eta$. Because $s(|\beta|-|\alpha|)+|\alpha|-\mu=0$ by assumption the right-hand side of (3.4) is bounded when $|\eta|$ tends to infinity. By (3.2), (3.3) and (3.4) we see that the map $x^{\alpha} \partial^{\beta}: G(\mu) \rightarrow G(0)$ is continuous.

Let $p(\eta)$ be a function on $\mathbb{N}^{2}$ such that

$$
|p(\eta)| \leq C|\eta|^{m}, \forall \eta \in \mathbb{N}^{2}
$$

for some $C>0$ and $m \geq 0$ independent of $\eta$. Then we define the Euler type pseudodifferential operator $p(\delta)$ on $G_{w}^{s}(\mu)$ by

$$
p(\delta) u:=\sum_{\eta} u_{\eta} p(\eta) x^{\eta} / \eta !, \quad u=\sum_{\eta} u_{\eta} x^{\eta} / \eta ! \in G_{w}^{s}(\mu)
$$

where we set $\delta=\left(\delta_{1}, \delta_{2}\right), \delta_{j}=x_{j}\left(\partial / \partial x_{j}\right), j=1,2$. We note that if $p(\eta)=\eta_{1}+\eta_{2}$, the operator $p(\delta)=\delta_{1}+\delta_{2}$ is a so-called Euler type differential operator. Then we have

Lemma 3.3. Let $p(\eta)$ be a function on $\mathbb{N}^{2}$ such that $\sup _{|\eta| \geq N}|p(\eta)| \rightarrow 0$ when $N \rightarrow \infty$. Then the map $p(\delta): G_{w}^{s}(\mu) \rightarrow G_{w}^{s}(\mu)$ is a compact operator for every $\mu \geq 0$.

The proof of this lemma follows exactly the same arguments of the former half of the proof of Lemma 3.2. Therefore we omit the proof.

In the following we give basic properties of Fredholm operators on a Hilbert space $H$ with norm $\|\cdot\|$. We denote by $\mathscr{L}(H)$ the space of linear con- 
tinuous operators on $H$. An operator $L \in \mathscr{L}(H)$ is said to be a Fredholm operator if the range $L H$ of $L$ is closed in $H$, and the kernel and cokernel of $L$ are of finite dimension, i.e., $\operatorname{dim} \operatorname{Ker} L<\infty$ and $\operatorname{dim} \operatorname{Coker} L<\infty$, where $\operatorname{Coker} L=H / L H$. We denote the set of Fredholm operators by $\Psi(H)$. For $L \in \Psi(H)$ an index of $L$ is defined by

$$
\text { ind } L:=\operatorname{dim} \operatorname{Ker} L-\operatorname{dim} \text { Coker } L \text {. }
$$

Let $C_{\infty}(H)$ be the space of compact operators on $H$, and let $I$ denote the identity operator on $H$. Then the following two lemmas are well known. (cf. [3]).

Lemma 3.4. An operator $L \in \mathscr{L}(H)$ is a Fredholm operator if and only if there exist linear continuous operators $R_{1} \in \mathscr{L}(H), R_{2} \in \mathscr{L}(H)$ and compact operators $K_{1} \in C_{\infty}(H), K_{2} \in C_{\infty}(H)$ such that

$$
R_{1} L=I+K_{1} . L R_{2}=I+K_{2}
$$

Here the operators $R_{1}$ and $R_{2}$ are called left and right regularizers, respectively.

Lemma 3.5. The set $\Psi(H)$ is an open subset of $\mathscr{L}(H)$ and the index is constant on each connected component of $\Psi(H)$. If $L \in \Psi(H)$ and $K \in C_{\infty}(H)$ the operator $L+K$ is in $\Psi(H)$ and ind $(L+K)=$ ind $L$.

Lemma 3.6. Let $L \in \mathscr{L}(H)$ and let $L^{*}$ denote the adjoint operator of $L$. Then $L \in \Psi(H)$ if and only if there exist a positive constant $A$, a positive integer $n$ and $K_{J}, \tilde{K}_{J} \in C_{\infty}(H),(j=1,2, \ldots, n)$ such that the following inequalities hold:

$$
\begin{aligned}
& \left\|L f i+\sum_{j=1}^{n}\right\| K, f\|\geq A\| f \|, \quad \forall f \in H, \\
& \left\|L^{*} f\right\|+\sum_{j=1}^{n}\|\tilde{K}, f\| \geq A\|f\|, \quad \forall f \in H .
\end{aligned}
$$

Moreover, if $L \in \Psi(H)$ we can take $n=1$ in (3.7) and (3.8).

Proof. Suppose that $L$ is a Fredholm operator. Let $H=H_{1} \oplus H_{2}$ and $H=$ $H_{1}^{\prime} \oplus H_{2}^{\prime}$ be direct decompositions of $H$, where $H_{1}=\operatorname{Ker} L, H_{1}^{\prime}=\operatorname{Coker} L$. We denote by $K$ the projection onto $H_{1}$. Clearly $K \in C_{\infty}(H)$ and $L: H_{2} \rightarrow H_{2}^{\prime}$ is continuous and bijective. Hence the closed graph theorem implies that $L$ is a homeomorphism. Therefore, if we write $f=f_{1}+f_{2} \in H_{1} \oplus H_{2}$ we have 


$$
\|L f\|=\left\|L f_{2}\right\| \geq A\left\|f_{2}\right\|=A\|f\|-A\left\|f_{1}\right\| \geq A\|f\|-\|K f\|
$$

for some $A>0$. This proves (3.7). The inequality (3.8) will be proved similarly because $L^{*}$ is also a Fredholm operator.

Conversely, let us suppose that (3.7) and (3.8) are satisfied. By (3.7) we have that $\sum_{j=1}^{n}\|K, f\| \geq A\|f\|$ if $f \in \operatorname{Ker} L$. Suppose that $\operatorname{Ker} L$ is of infinite dimension. Then we can find a sequence $f_{k} \in \operatorname{Ker} L$ such that $\left\|f_{k}\right\|=1$ and $f_{k}$ weakly converges to zero when $k \rightarrow \infty$. Because $K_{j}$ 's are compact, the sequence $K_{j} f_{k}$ strongly converges to zero when $k \rightarrow \infty$ for each $j$. This contradicts to the above inequality. Therefore it follows that $\mathrm{Ker} L$ is of finite dimension. Similarly (3.8) implies that $\operatorname{Ker} L^{*}$ is of finite dimension.

In order to show that the range of $L$ is closed, we first show the following

(*) Every bounded sequence $f_{k} \in H$ such that $L f_{k}$ is strongly convergent has a strongly convergent subsequence.

Indeed, $f_{k}$ contains a weakly convergent subsequence because $f_{k}$ is bounded. Without loss of generality, we may assume that $f_{k}$ weakly converges to some $f$ $\in H$. By the continuity of $L$ it follows that $L f_{k}$ weakly converges to $L f$. Because $L f_{k}$ strongly converges by assumption, it follows that $L f_{k}$ strongly converges to $L f$. On the other hand, by the compactness of $K_{j}$ in (3.7) the sequence $K_{f} f_{k}$ strongly converges to $K, f$ when $k \rightarrow \infty$. By (3.7) with $f$ replaced by $f_{k}-f$ we see that the sequence $\left\{f_{k}\right\}$ strongly converges to $f$. Hence we have the assertion.

Let $H_{2}$ be an orthogonal complement of $\operatorname{Ker} L=: H_{1}$. It then follows that

$$
\|f\| \leq C\|L f\|, \quad f \in H_{2},
$$

for some $C>0$. In fact, otherwise there is a sequence $f_{k} \in H_{2}$ with $\left\|f_{k}\right\|=1$ and $\left\|L f_{k}\right\| \leq 1 / k$. By the property $(*), f_{k}$ contains a subsequence strongly convergent to $f$ such that $f \in H_{2}$ with $\|f\|_{1}=1, L f=0$, which is a contradiction proving the assertion. The closedness of the range of $L$ is clear from (3.10). Since $L H=\overline{L H}=\operatorname{Ker} L^{*}{ }^{1}$, it follows that Coker $L$ is of finite dimension. 圈

\section{\$4. Reduction to Toeplitz Operators}

We set $\langle\eta\rangle:=\left(1+|\eta|^{2}\right)^{1 / 2}$ and we denote by $\langle\delta\rangle$ the Euler type pseudodifferential operator with symbol $\langle\eta\rangle$ : Let $P$ be given by (2.3) and let $m$ be an $s$-Gevrey order of $P$. Then we have 
Proposition 4.1. Let the operators $P_{0}$ and $Q_{0}$ be defined by

$$
Q_{0}:=P_{0}\langle\delta\rangle^{-m}, \quad P_{0}(x, \partial):=\sum_{|\beta|+(1-s)(|\alpha|-|\beta|)=m} a_{\alpha \beta} \partial^{\beta} x^{\alpha}
$$

Then $Q_{0}$ maps $G_{w}^{s}(0)$ into itself. Moreover $Q_{0}$ is a Fredholm operator if and only if $P: G_{w}^{s}(m) \rightarrow G_{w}^{s}(0)$ is a Fredholm operator.

Proof. In the following, we omit the suffices of $G_{w}^{s}(\mu)$ and write it by $G(\mu)$. We write the operator $P$ in the following form

$$
\begin{aligned}
P(x, \partial) & =\sum_{|\beta|+(1-s)(|\alpha|-|\beta|)=m} a_{\alpha \beta} \partial^{\beta} x^{\alpha}+\sum_{|\beta|+(1-s)(|\alpha|-|\beta|)<m} a_{\alpha \beta} \partial^{\beta} x^{\alpha} \\
& =: P_{0}(x, \partial)+P_{1}(x, \partial) .
\end{aligned}
$$

Because the $s^{-}$Gevrey order of each term $\partial^{\beta} x^{\alpha}$ in $P_{1}$ is smaller than $m$, it follows from Lemmas 3.1 and 3.2 that the map $P_{1}: G(m) \rightarrow G(0)$ is compact. Therefore by Lemma 3.5 it follows that $P_{0}: G(m) \rightarrow G(0)$ is a Fredholm operator if and only if $P: G(m) \rightarrow G(0)$ is a Fredholm operator.

Since we can easily see that $\langle\delta\rangle^{-m}: G(0) \rightarrow G(m)$ is an isomorphism, we conclude that $Q_{0}: G(0) \rightarrow G(0)$ is a Fredholm operator if and only if $P_{0}: G(m)$ $\rightarrow G(0)$ is a Fredholm operator.

Next we shall show that the Fredholmness of the operator $Q_{0}: G_{w}^{s}(0) \rightarrow$ $G_{w}^{s}(0)$ is equivalent to that of a certain Toeplitz operator on the two dimensional torus $\mathbb{T}^{2}$. Let us take the coordinate $\left(e^{i \theta_{1}}, e^{i \theta_{2}}\right) \in \mathbb{T}^{2}$. Let $u=\sum u_{n} x^{\eta} / \eta$ ! $\in G_{w}^{s}(0)$. We set $v_{\eta}:=u_{\eta} w^{\eta} /|\eta| !^{s}$. Then $u \in G_{w}^{s}(0)$ if and only if the sequence $\left\{v_{\eta}\right\}$ is in $\ell_{2}^{+}:=\ell_{2}\left(\mathbb{Z}_{+}^{2}\right)$, the set of square summable sequences on $\mathbb{Z}_{+}^{2}$, where $\mathbb{Z}_{+}$is the set of nonnegative integers. Because the space $\ell_{2}^{+}$and the Hardy space $H^{2}\left(\mathbb{T}^{2}\right)$ are isomorphic, it follows that $u \in G_{w}^{s}(0)$ if and only if $\sum_{\eta} v_{\eta} e^{i \theta \eta}$ is in $H^{2}\left(\mathbf{T}^{2}\right)$. Because $H^{2}\left(\mathbf{T}^{2}\right)$ is a closed subspace of $L^{2}\left(\mathbf{T}^{2}\right)$, the space of square integrable functions, there is a projection $\pi$ from $L^{2}\left(\mathbf{T}^{2}\right)$ onto $H^{2}\left(\mathbf{T}^{2}\right)$. By the correspondence between the spaces $G_{w}^{s}(0)$ and $H^{2}\left(\mathbf{T}^{2}\right)$ the Euler type operator $p(\delta)$ in (3.6) on $G_{w}^{s}(0)$ also defines a pseudodifferential operator $p\left(D_{\theta}\right)\left(D_{\theta}=i^{-1} \partial / \partial \theta\right)$ on $H^{2}\left(\mathbb{T}^{2}\right)$. We denote by $\lambda_{\alpha}\left(D_{\theta}\right)$ the pseudodifferential operator with symbol $\lambda_{\alpha}(\eta):=\eta^{\alpha}|\eta|^{-|\alpha|}(\eta \neq 0)$ and $\lambda_{\alpha}(0)=0$. We define a Toeplitz operator on $H^{2}\left(\mathbf{T}^{2}\right)$ by 


$$
T=\pi \sum_{|\beta|+(1-s)(|\alpha|-|\beta|)=m} a_{\alpha \beta} w^{\alpha-\beta} e^{i(\alpha-\beta) \theta} \lambda_{\alpha}\left(D_{\theta}\right): H^{2}\left(\mathbb{T}^{2}\right) \rightarrow H^{2}\left(\mathbb{T}^{2}\right)
$$

We note that, by $(2.5), T=\pi L\left(e^{\imath \theta}, D_{\theta} /\left|D_{\theta}\right|\right)$.

Proposition 4.2. The operator $Q_{0}: G_{w}^{s}(0) \rightarrow G_{w}^{s}(0)$ is a Fredholm operator if and only if the Toeplitz operator $T: H^{2}\left(\mathbb{T}^{2}\right) \rightarrow H^{2}\left(\mathbb{T}^{2}\right)$ is a Fredholm operator.

Proof. By the isomorphism between $G_{w}^{s}(0)$ and $H^{2}\left(\mathbb{T}^{2}\right)$ the projection $\pi$ : $L^{2}\left(\mathbb{T}^{2}\right) \rightarrow H^{2}\left(\mathbb{T}^{2}\right)$ naturally induces a projection on the formal Laurent series

$$
\pi u:=\sum_{\eta \in \mathbb{Z}_{+}^{2}} u_{\eta} x^{\eta} / \eta ! \text { for } u=\sum_{\eta \in \mathbb{Z}^{2}} u_{n} x^{\eta} / \eta !
$$

Here we use the same notation $\pi$ for the sake of simplicity. We note that, for $k$ $\geq 0, n \geq 0$ and $m \geq 0$

$$
\begin{aligned}
\left(\frac{\partial}{\partial t}\right)^{k} t^{m} t^{n} & =(n+m)(n+m-1) \cdots(n+m-k+1) t^{n+m-k} \\
& = \begin{cases}t^{n+m-k} \frac{\Gamma(n+m+1)}{\Gamma(n+m-k+1)}, & \text { if } n+m-k \geq 0 \\
0 & \text { if } n+m-k<0\end{cases}
\end{aligned}
$$

where $\Gamma$ denotes the gamma function. Similarly, if $k<0$ we have

$$
\left(\frac{\partial}{\partial t}\right)^{k} t^{m} t^{n}=\frac{t^{n+m-k}}{(n+m+1) \cdots(n+m-k)}=t^{n+m-k} \frac{\Gamma(n+m+1)}{\Gamma(n+m-k+1)}
$$

If we define Euler type operator $p_{\alpha \beta}(\delta)$ on $G(\mu)(\mu \geq 0)$ by

$$
p_{\alpha \beta}(\eta)= \begin{cases}\prod_{j=1}^{2} \frac{\Gamma\left(\eta_{j}+\alpha_{j}+1\right)}{\Gamma\left(\eta, \alpha_{j}-\beta_{j}+1\right)}, & \text { if } \eta+\alpha-\beta \in \mathbb{Z}_{+}^{2} \\ 0 & \text { if } \eta+\alpha-\beta \notin \mathbb{Z}_{+}^{2}\end{cases}
$$

we have, for $\alpha \in \mathbb{Z}_{+}^{2}$ and $\beta \in \mathbb{Z}^{2}$

$$
\partial^{\beta} x^{\alpha} u=x^{\alpha-\beta} p_{\alpha \beta}(\delta) u, \text { for } u \in G_{w}^{s}(\mu)(\mu \geq 0)
$$


Therefore we have that

$$
P_{0}(x, \partial)=\sum_{|\beta|+(1-s)(|\alpha|-|\beta|)=m} a_{\alpha \beta} x^{\alpha-\beta} p_{\alpha \beta}(\delta)
$$

By the definition of $p_{\alpha \beta}(\delta)$ in (4.6) we see that in the expression of $x^{\alpha-\beta} p_{\alpha \beta}$ $(\delta)\langle\delta\rangle^{-m} u(u \in G(0))$ there appear no negative powers. Hence we have

$$
\begin{aligned}
Q_{0} & =\pi \sum a_{\alpha \beta} x^{\alpha-\beta} p_{\alpha \beta}(\delta)\langle\delta\rangle^{-m} \\
& =\pi \sum a_{\alpha \beta} x^{\alpha-\beta}\langle\delta\rangle^{(s-1)(|\alpha|-|\beta|)}\langle\delta\rangle^{-|\beta|} p_{\alpha \beta}(\delta)
\end{aligned}
$$

on $G(0)$.

We shall study the operators $\pi x^{\gamma}\langle\delta\rangle^{(s-1)|\gamma|}(\gamma=\alpha-\beta)$ and $\langle\delta\rangle^{-|\beta|} p_{\alpha \beta}(\delta)$. Let $\imath=\sum u_{\eta} x^{\eta} / \eta ! \in G(0)$. We set $v_{\eta}:=u_{\eta} w^{\eta} /|\eta| !$. Then we have

$$
\begin{aligned}
\pi x^{r}\langle\delta\rangle^{(s-1)|\gamma|} \sum u_{\eta} \frac{x^{\eta}}{\eta !} & =\pi x^{r}\langle\delta\rangle^{(s-1)|\gamma|} \sum v_{\eta} w^{-\eta}|\eta| !^{s} \frac{x^{\eta}}{\eta !} \\
& =\sum_{\eta+r \geq 0, \eta \geq 0} v_{\eta} w^{-\eta}|\eta| !^{s}\langle\eta\rangle^{(s-1)|r|} \frac{x^{\eta+\gamma}}{\eta !} \\
& =\sum v_{\eta-\gamma} w^{\gamma-\eta}(|\eta|-|\gamma|) !^{s}\langle\eta-\gamma\rangle^{(s-1)|\gamma|} \frac{\eta !}{(\eta-\gamma) !} \frac{x^{\eta}}{\eta !}
\end{aligned}
$$

Therefore $\pi x^{r}\langle\delta\rangle^{(s-1)|r|}$ is given by

$$
\pi x^{r}\langle\delta\rangle^{(s-1)|r|}:\left\{v_{\eta}\right\} \in \ell_{2}^{+} \rightarrow\left\{v_{\eta-\gamma} w^{\gamma} \frac{(|\eta|-|\gamma|) !^{s}}{|\eta| !^{s}}\langle\eta-\gamma\rangle^{(s-1) \mid r i} \frac{\eta !}{(\eta-\gamma) !}\right\} \in \ell_{2}^{+} .
$$

We define the pseudodifferential operator $A_{r}\left(D_{\theta}\right)$ with symbol $A_{r}(\eta)$ by

$$
A_{\gamma}(\eta):=\frac{|\eta| !^{s}\langle\eta\rangle(s-1)|\gamma|}{(|\eta|+|\gamma|) !^{s}} \frac{(\eta+\gamma) !}{\eta !}
$$

Let $S_{\gamma}$ be a multiplication operator by a function $e^{i \gamma \theta}, S_{\gamma} \sum u_{\eta} e^{i \eta \theta}=\sum u_{\eta} e^{\mathrm{i}(\eta+\gamma) \theta}$. Then it follows from (4.9) that the operator $\pi x^{r}\langle\delta\rangle^{(s-1)|r|}$ corresponds to

$$
\pi S_{r} A_{r}\left(D_{\theta}\right) w^{\gamma}
$$


We have, assuming $\eta+\gamma \geq 0$

$$
\frac{|\eta| !^{s}\langle\eta\rangle^{(s-1)|r|}}{(|\eta|+|\gamma|) ! !^{s}} \frac{(\eta+\gamma) !}{\eta !}=\widetilde{\lambda}_{r}(\eta)+r_{r}(\eta), \tilde{\lambda}_{r}(\eta)=\frac{\left(\eta_{1}+1\right)^{r_{1}}\left(\eta_{2}+1\right)^{r^{2}}}{\langle\eta\rangle^{|r|}}
$$

where $r_{\gamma}(\eta)$ consists of terms such that $r_{\gamma}(\eta) \rightarrow 0$ when $|\eta| \rightarrow \infty$.

Indeed the quantity $|\eta| !^{s}\langle\eta\rangle^{s|\gamma|}(|\eta|+|\gamma|) !^{-s}$ tends to 1 when $|\eta| \rightarrow \infty$ and $\gamma$ fixed. On the other hand, we get, assuming $\eta_{j}+\gamma_{j} \geq 0$,

$$
\frac{\left(\eta_{j}+\gamma_{j}\right) !}{\eta_{j} !}\langle\eta\rangle^{-r}=\left(\frac{\eta_{j}+1}{\langle\eta\rangle}\right)^{r}+\Psi_{j}(\eta)
$$

with $\Psi_{j}(\eta)$ satisfying $\Psi_{j}(\eta) \rightarrow 0$ when $|\eta| \rightarrow \infty$. By these estimates we have (4.12).

It follows from (4.11), (4.12) and the definition of $\pi$ that

$$
\pi S_{r} A_{r}\left(D_{\theta}\right) w^{\gamma}=\pi S_{r} \widetilde{\lambda}_{r}\left(D_{\theta}\right) w^{\gamma}+\pi R_{r}\left(D_{\theta}\right)
$$

where $R_{\gamma}\left(D_{\theta}\right):=S_{\gamma} r_{\gamma}\left(D_{\theta}\right) w^{\gamma}$, with $\gamma_{\gamma}\left(D_{\theta}\right)$ being the psedodifferential operator with symbol $r_{\gamma}(\eta)$. We note that by Lemma 3.3 the operator $\pi R_{\gamma}\left(D_{\theta}\right)$ is a compact operator.

Next we consider the operator $\langle\delta\rangle^{-|\beta|} p_{\alpha \beta}(\delta)$. In view of the relation

$$
\langle\delta\rangle^{-|\beta|} p_{\alpha \beta}(\delta) \sum_{\eta \geq 0} u_{\eta} \frac{x^{\eta}}{\eta !}=\sum_{\eta \geq \beta-\alpha} v_{\eta} w^{-\eta}|\eta|^{s}\langle\eta\rangle^{-|\beta|} p_{\alpha \beta}(\eta) \frac{x^{\eta}}{\eta !} .
$$

the operator $\langle\delta\rangle^{-|\beta|} p_{\alpha \beta}(\delta)$ corresponds to the pseudodifferential operator $\left\langle D_{\theta}\right\rangle^{-|\beta|} p_{\alpha \beta}\left(D_{\theta}\right)$ on $H^{2}$. If $\eta+\alpha-\beta \geq 0$ we have

$$
\langle\eta\rangle^{-|\beta|} p_{\alpha \beta}(\eta)=\tilde{\lambda}_{\beta}(\eta)+\tilde{r}_{\alpha \beta}(\eta)
$$

where $\tilde{r}_{\alpha \beta}(\eta)$ satisfies that $\sup _{|\eta| \geq n}\left|\tilde{r}_{\alpha \beta}(\eta)\right| \rightarrow 0$ when $n$ tends to infinity. Therefore the operator $\langle\delta\rangle^{-|\beta|} p_{\alpha \beta}(\delta)$ in (4.8) corresponds to $\widetilde{\lambda}_{\beta}\left(D_{\theta}\right)+$ $\tilde{\gamma}_{\alpha \beta}\left(D_{\theta}\right)$.

By (4.14) with $\gamma=\alpha-\beta$ we get from (4.8) that $Q_{0}$ corresponds to the operator

$$
\pi \sum_{\alpha \beta}\left(S_{\alpha-\beta} \tilde{\lambda}_{\alpha-\beta} w^{\alpha-\beta}+R_{\alpha-\beta}\right)\left(\tilde{\lambda}_{\beta}+\tilde{\gamma}_{\alpha \beta}\right)
$$




$$
\begin{aligned}
& =\pi \sum a_{\alpha \beta} S_{\alpha-\beta} w^{\alpha-\beta} \tilde{\lambda}_{\alpha-\beta} \tilde{\lambda}_{\beta}+\pi \sum a_{\alpha \beta} K_{\alpha \beta} \\
& =\pi \sum a_{\alpha \beta} S_{\alpha-\beta} \tilde{\lambda}_{\alpha} w^{\alpha-\beta}+\pi \sum a_{\alpha \beta} K_{\alpha \beta},
\end{aligned}
$$

where

$$
K_{\alpha \beta}=S_{\alpha-\beta} \tilde{\lambda}_{\alpha-\beta} w^{\alpha-\beta} \tilde{\gamma}_{\alpha \beta}+R_{\alpha-\beta} \tilde{\lambda}_{\beta}+R_{\alpha-\beta} \tilde{r}_{\alpha \beta} .
$$

For each $\alpha$ and $\beta, K_{\alpha \beta}$ is a compact operator by the definition of symbols $R_{\alpha-\beta}$ and $\tilde{\gamma}_{\alpha \beta}$ and Lemma 3.3. Since the sum $\sum a_{\alpha \beta} K_{\alpha \beta}$ is a finite sum, the second term in the right-hand side of (4.15) is a compact operator. Because $\tilde{\lambda}_{\alpha}-$ $\eta^{\alpha} /|\eta|^{|\alpha|}$ defines a compact pseudodifferential operator on $H^{2}$ the right-hand side of (4.15) is equal to $T$ modulo compact operators.

In view of Propositions 4.1 and 4.2 it is sufficient to show the following theorem.

Theorem 4.3. Suppose that the Toeplitz operator $T: H^{2}\left(\mathbf{T}^{2}\right) \rightarrow H^{2}\left(\mathbf{T}^{2}\right)$ in (4.3) is a Fredholm operator. Then the conditions (2.6) and (2.7) are necessary.

We shall prove this theorem in Sections 5 and 6 .

\section{\$5. Proof of Theorem 4.3. - the Necessity of (2.6)}

In this section we shall prove the necessity of (2.6) in Theorem 4.3. We use the same notations as in the previous sections unless otherwise is stated. We define the projections $\pi_{1}$ and $\pi_{2}$ by

$$
\pi_{j} u=\sum_{\eta, \geq 0} u_{\eta} e^{i \eta \theta}, \quad \text { for } u=\sum_{\eta} u_{\eta} e^{i \eta \theta} \in L^{2}\left(\mathbf{T}^{2}\right), \quad(j=1,2)
$$

Then the spaces $H_{j}^{2}$,

$$
H_{j}^{2} \equiv H_{j}^{2}\left(\mathbf{T}^{2}\right):=\pi_{j} L^{2}\left(\mathbf{T}^{2}\right), \quad(j=1,2),
$$

are closed subspaces of $L^{2}\left(\mathbf{T}^{2}\right)$. It is obvious that $\pi=\pi_{1} \pi_{2}$. Recall that $L(z$, $\xi)$ denotes the Toeplitz symbol defined in (2.5). Let $\mathscr{L}\left(\theta, D_{\theta}\right)$ be the pseudo- 
differential operator of order zero on $\mathbb{T}^{2}$ with symbol $L(z, \xi /|\xi|)$ where $z=$ $\left(e^{i \theta_{1}}, e^{i \theta_{2}}\right)$. Then it follows from (4.3) that

$$
T=\pi \mathscr{L}\left(\theta, D_{\theta}\right) ; H^{2}\left(\mathbb{T}^{2}\right) \rightarrow H^{2}\left(\mathbb{T}^{2}\right)
$$

We define Toeplitz operators $T_{j}(j=1,2)$ by

$$
T_{j}:=\pi_{j} \mathscr{L}\left(\theta, D_{\theta}\right) ; H_{j}^{2}\left(\mathbb{T}^{2}\right) \rightarrow H_{j}^{2}\left(\mathbb{T}^{2}\right),
$$

and the multiplication operators $U_{j}(j=1,2)$ by

$$
U_{j} u:=\sum_{\eta} u_{\eta} e^{\imath \eta \theta+\imath \theta_{1}+\imath \theta_{2}-\imath \theta}, \quad \text { for } \quad u=\sum_{\eta} u_{\eta} e^{\imath \eta \theta} \in L^{2}\left(\mathbb{T}^{2}\right)
$$

Clearly, $U$, are unitary operators on $L^{2}$ and $\left[U_{1}, U_{2}\right]=0$, where $[A, B]:=A B$ $-B A$. Moreover we have that $\left[U_{j}, \pi_{j}\right]=0$, which is crucial in the following arguments.

For the later use we introduce the function class $\mathscr{A}_{\xi, \hat{\theta}}$ in the following way. Let $\xi \in \mathbb{R}_{+}^{2}, \xi \neq 0, \kappa>0$ and $\widehat{\theta}=\left(\widehat{\theta}_{1}, \widehat{\theta}_{2}\right), 0 \leq \widehat{\theta}_{j} \leq 2 \pi(j=1,2)$ are given. For $\varphi \in C^{\infty}\left(\mathbb{T}^{2}\right)$ with support contained in a small neighborhood of the origin we define

$$
\mathscr{A}_{\xi, \hat{\theta}}:=\left\{\varphi\left(\frac{\theta-\bar{\theta}}{\kappa}\right) e^{\imath \theta k \xi} ; \kappa \in \mathbb{N}\right\}
$$

Then we have the following

Lemma 5.1. Let $\xi$ be given by (5.5). Then under the same assumptions as in Theorem 4.3 there exist a constant $C>0$ and a psendodifferential operator $\mathscr{K}$ of order -1 on $\mathbb{T}^{2}$ such that

$$
\|\mathscr{L} g\|+\|\mathscr{K} g\| \geq C\|g\| \quad \text { for any } \quad \forall g \in \mathscr{A}_{\xi, \hat{\theta}}
$$

Proof. We shall divide the proof into 6 steps.

Step 1. By assumption there exist a compact operator $K$ on $H^{2}$ and $A>0$ such that

$$
\|T u\|+\|K u\| \geq A\|u\|, \quad \forall u \in H^{2}\left(\mathbb{T}^{2}\right) .
$$

We put $u=\pi g=\pi_{1} \pi_{2} g, g \in H_{1}^{2}$ and $Q_{j}=I-\pi_{j}(j=1,2)$ in (5.7). Because $\pi_{1} g=g$ for $g \in H_{1}^{2}$, we have, 


$$
\left\|\pi \mathscr{L}\left(\theta, D_{\theta}\right) \pi g\right\|+\|\pi K \pi g\|+A\left\|Q_{2} g\right\| \geq A\|g\|, \quad \forall g \in H_{1}^{2} .
$$

For a positive integer $\imath$ we get, from (5.8) that

$$
\left\|\pi \mathscr{L}\left(\theta, D_{\theta}\right) \pi U_{1}^{n} g\right\|+\left\|\pi K \pi U_{1}^{n} g\right\|+A\left\|Q_{2} U_{1}^{n} g\right\| \geq A\left\|U_{1}^{n} g\right\|, \quad \forall g \in H_{1}^{2} .
$$

Because $U_{1}$ is an isometry on $H_{1}^{2}$ we have

$$
\left\|U_{1}^{-n} \pi \mathscr{L}\left(\theta, D_{\theta}\right) \pi U_{1}^{n} g\right\|+\left\|\pi K \pi U_{1}^{n} g\right\|+A\left\|_{1}^{\mid} U_{1}^{-n} Q_{2} U_{1}^{n} g\right\| \geq A\|g\|, \forall g \in H_{1}^{2} .
$$

Similarly we replace $g$ in (5.10) with $\pi_{1} U_{2}^{n} g\left(g \in L^{2}\right)$. Then, by noting that $Q_{1}=I-\pi_{1}$ we have

$$
\begin{gathered}
\left\|U_{1}^{-n} \pi_{1} \pi_{2} \mathscr{L} \pi_{1} \pi_{2} U_{1}^{n} \pi_{1} U_{2}^{n} g\right\| \\
+\left\|\pi K \pi U_{1}^{n} \pi_{1} U_{2}^{n} g\right\|+A\left\|U_{1}^{-n} Q_{2} U_{1}^{n} \pi_{1} U_{2}^{n} g\right\| \\
+A\left\|Q_{1} U_{2}^{n} g\right\| \geq A_{1}^{\prime} \mid U_{2}^{n} g \|, \quad \forall g \in L^{2} .
\end{gathered}
$$

Step 2. We shall rewrite (5.11). We define the operators $V_{\jmath, n}(j=1,2)$ by

$$
V_{1, n}:=U_{1}^{-n} \pi_{2} U_{1}^{n}, \quad V_{2, n}:=U_{2}^{-n} \pi_{1} U_{2}^{n}
$$

Because $\left[U_{j}, \pi_{\jmath}\right]=0,\left[U_{1}, U_{2}\right]=0$ on $L^{2}$, we have $\left[V_{2, n}, U_{1}^{ \pm n}\right]=0$ and $\left[V_{1, n}\right.$, $\left.U_{2}^{ \pm n}\right]=0$ for every integer $n$. In addition it is easy to see that $\left[V_{1, n}, V_{2, n}\right]=$ 0 and $\left(V_{\jmath, n}\right)^{2}=V_{\jmath, n}$ for $j=1.2$. It follows that

$$
\begin{aligned}
\left\|U_{1}^{-n} \pi_{1} \pi_{2} \mathscr{L}_{1} \pi_{2} U_{1}^{n} \pi_{1} U_{2}^{n} g\right\| & =\left\|U_{2}^{-n} U_{1}^{-n} \pi_{1} \pi_{2} \mathscr{L} \pi_{1} \pi_{2} U_{1}^{n} \pi_{1} U_{2}^{n} g\right\| \\
& =\left\|V_{1, n} U_{1}^{-n} V_{2, n} U_{2}^{-n} \mathscr{L} U_{2}^{n} V_{2, n} U_{2}^{-n} U_{1}^{n} V_{1, n} U_{2}^{n} V_{2, n} g\right\| \\
& =\left\|V_{1, n} V_{2, n} U_{1}^{-n} U_{2}^{-n} \mathscr{L} U_{2}^{n} U_{1}^{n} V_{1, n} V_{2, n} g\right\| .
\end{aligned}
$$

In the same way we have

$$
\left\|\pi K \pi U_{1}^{n} \pi_{1} U_{2}^{n} g\right\|=\left\|\pi_{1} \pi_{2} K U_{1}^{n} U_{2}^{n} V_{1, n} V_{2, n} g\right\|
$$

$$
\begin{gathered}
\left\|U_{1}^{-n} Q_{2} U_{1}^{n} \pi_{1} U_{2}^{n} g\right\|=\left\|U_{2}^{-n} U_{1}^{-n} Q_{2} U_{1}^{n} \pi_{1} U_{2}^{n} g\right\|=\left\|\left(I-V_{1, n}\right) V_{2, n} g\right\|, \\
\left\|Q_{1} U_{2}^{n} g\right\|=\left\|U_{2}^{-n} Q_{1} U_{2}^{n} g\right\|=\left\|\left(I-V_{2, n}\right) g\right\| .
\end{gathered}
$$


By inserting (5.13) - (5.16) to (5.11) we obtain the following

$$
\begin{aligned}
& \left\|V_{1, n} V_{2, n} U_{1}^{-n} U_{2}^{-n} \mathscr{L} U_{2}^{n} U_{1}^{n} V_{1, n} V_{2, n} g\right\|+\left\|\pi_{1} \pi_{2} K U_{1}^{n} U_{2}^{n} V_{1, n} V_{2, n} g\right\| \\
& +A\left\|\left(I-V_{1, n}\right) V_{2, n} g\right\|+A\left\|\left(I-V_{2, n}\right) g\right\| \geq A\|g\|, \quad \forall g \in L^{2} .
\end{aligned}
$$

Step 3. We shall estimate the limit when $n \rightarrow \infty$ in (5.17) for $g \in \mathscr{A}_{\xi, \hat{\theta} \text {. }}$. To this end, we first calculate the Fourier coefficients of $g(\theta):=\varphi((\theta-\hat{\theta})$ $/ \kappa) e^{i \theta k \xi}$. Because the support of $\varphi$ is contained in some neighborhood of the origin, the support of the function $\varphi((\theta-\widehat{\theta}) / \kappa)$ is contained in a neighborhood of $\widehat{\theta}$ if we take $\kappa>0$ sufficiently small. Therefore, if $\kappa$ is sufficiently small the Fourier coefficient $\hat{g}(\eta)$ of $g(\theta)$ is given by

$$
\int_{\mathbb{T}^{2}} e^{-\imath \theta n} \varphi\left(\frac{\theta-\bar{\theta}}{\kappa}\right) e^{i \theta k \xi} d \theta=\int_{\mathbb{R}^{2}} e^{-i(y \kappa+\theta)(\eta-k \xi)} \kappa^{2} \varphi(y) d y=e^{-i \theta(\eta-k \xi)} \kappa^{2} \widehat{\varphi}((\eta-k \xi) \kappa)
$$

where $\widehat{\varphi}$ denotes the Fourier transform of $\varphi$. It follows that the right-hand side is rapidly decreasing in $\eta$ when $\eta \rightarrow \infty$ in a sufficiently small conical neighborhood of $\xi_{0}$ such that $\xi_{0} \neq \xi$ uniformly in $k$.

By the definition of $V_{2, n}$ in (5.12) we have

$$
V_{2, n} g=\sum_{\eta_{1} \geq-n} e^{i \eta \theta-i(\eta-k \xi) \hat{\theta}} \kappa^{2} \widehat{\varphi}((\eta-k \xi) \kappa), g=\varphi((\theta-\widehat{\theta}) / \kappa) e^{i \theta k \xi} \in \mathscr{A}_{\xi, \hat{\theta}}
$$

Therefore we have

$$
\left\|\left(I-V_{2, n}\right) g\right\| \rightarrow 0(n \rightarrow \infty)
$$

where the limit is uniform with respect to $k,(k=1,2, \ldots)$.

Similarly, by simple computations we have

$$
\left(I-V_{1, n}\right) V_{2, n} g=\sum_{\eta_{1} \geq-n, \eta_{2}<-n} e^{i \eta \theta-i \hat{\theta}(\eta-k \xi)} \kappa^{2} \bar{\varphi}((\eta-k \xi) \kappa), g=\varphi\left(\frac{\theta-\hat{\theta}}{\kappa}\right) e^{i \theta k \xi} \in \mathscr{A}_{\xi, \hat{\theta}}
$$

It follows that

$$
\left\|\left(I-V_{1, n}\right) V_{2, n} g\right\| \rightarrow 0(n \rightarrow \infty),
$$

uniformly with respect to $k$. 
Step 4. We shall estimate the term $\left\|\pi_{1} \pi_{2} K U_{1}^{n} U_{2}^{n} V_{1, n} V_{2, n} g\right\|$ in (5.17) when $n \rightarrow \infty$. We note

$$
V_{1, n} V_{2, n} g=\sum_{\eta_{1} \geq-n, \eta_{2} \geq-n} e^{i \eta \theta-i(\eta-k \xi) \hat{\theta}} \kappa^{2} \hat{\varphi}((\eta-k \xi) \kappa)
$$

for $g=\varphi((\theta-\hat{\theta}) / \kappa) e^{i \theta k \xi} \in \mathscr{A}_{\xi, \hat{\theta}}$. It follows that

$$
\lim _{n \rightarrow \infty}\left\|V_{1, n} V_{2, n} g-g\right\|=0 \quad \text { uniformly in } \quad g \in \mathscr{A}_{\xi, \hat{\theta}}
$$

By (5.22) and the definitions of $\mathscr{A}_{\xi, \hat{\theta}}, U_{1}$ and $U_{2}$ we easily see that $U_{1}^{n} U_{2}^{n} V_{1, n}$ $V_{2, n} g$ weakly converges to zero uniformly in $g \in \mathscr{A}_{\xi, \hat{\theta}}$ when $n \rightarrow \infty$. In order to see this it is sufficient to show that $U_{1}^{n} U_{2}^{n} g$ weakly converges to zero uniformly in $g \in \mathscr{A}_{\xi, \hat{\theta}}$ when $n \rightarrow \infty$. This follows from the definition of $U_{1}$ and $U_{2}$ in (5.4).

We want to show that $K U_{1}^{n} U_{2}^{n} V_{1, n} V_{2, n} g$ converges to zero strongly and uniformly on $\mathscr{A}_{\xi, \hat{\theta}}$ when $n \rightarrow \infty$. Let $\varepsilon>0$ be arbitrarily given. Because the set $B$ $:=\left\{U_{1}^{n} U_{2}^{n} V_{1, n} V_{2, n} g ; n=1,2, \ldots, g \in \mathscr{A}_{\xi, \hat{\theta}}\right\}$ is bounded in $L^{2}$, the set $K B$ is precom. pact. Thus we can find a finite $g_{j} \in \mathscr{A}_{\xi, \hat{\theta}}$ and integers $n_{j}(j=1, \ldots, \ell)$ such that the set $K B$ is covered by $\varepsilon$-neighborhoods of $h_{j}:=K U_{1}^{n} U_{2}^{n} V_{1, n} V_{2, n} g\left(n=n_{j}, j\right.$ $=1, \ldots, \ell)$. Let us write $h_{j}=\sum_{\eta} h_{j}(\eta) e^{\imath \eta \theta}$ and let $N \gg 1$. For every $h \in K B, h$ $=\sum_{\eta} h(\eta) e^{\imath \eta \theta}$ we can find $j$ such that $\left\|h-h_{j}\right\|<\varepsilon$. It follows that

$$
\begin{aligned}
\left(\sum_{|\eta| \geq N}|h(\eta)|^{2}\right)^{1 / 2} & \leq\left(\sum_{|\eta| \geq N}\left|h(\eta)-h_{j}(\eta)\right|^{2}\right)^{1 / 2}+\left(\sum_{|\eta| \geq N}\left|h_{\jmath}(\eta)\right|^{2}\right)^{1 / 2} \\
& \leq \varepsilon+\left(\sum_{|\eta| \geq N}\left|h_{j}(\eta)\right|^{2}\right)^{1 / 2} \rightarrow \varepsilon(N \rightarrow \infty)
\end{aligned}
$$

Thus there exists $N \geq 1$ such that

$$
\left(\sum_{|\eta| \geq N}|h(\eta)|^{2}\right)^{1 / 2} \leq 2 \varepsilon \text { for all } \forall h \in K B
$$

For such an integer $N$ we take a cutoff function $\phi(\eta)$ supported on $|\eta|<N$ such that $\phi \equiv 1$ on $|\eta|<N, \eta \in \mathbb{Z}^{2}$. Then for the pseudodifferential operator $\phi \equiv$ $\phi\left(D_{\theta}\right)$ we have

$$
K U_{1}^{n} U_{2}^{n} V_{1, n} V_{2, n} g=\phi K U_{1}^{n} U_{2}^{n} V_{1, n} V_{2, n} g+(1-\phi) K U_{1}^{n} U_{2}^{n} V_{1, n} V_{2, n} g
$$


The second term in the right-hand side is smaller than $2 \varepsilon$. As to the first term we note that it strongly converges to zero uniformly with respect to $g \in \mathscr{A}_{\xi, \hat{\theta}}$ when $n \rightarrow \infty$ if and only if $K U_{1}^{n} U_{2}^{n} V_{1, n} V_{2, n} g$ weakly converges to zero uniformly with respect to $g \in \mathscr{A}_{\xi, \hat{\theta}}$. In view of the arguments in the above, we have the latter assertion.

Step 5. We shall estimate $\left\|V_{1, n} V_{2, n} U_{1}^{-n} U_{2}^{-n} \mathscr{L}_{U_{1}^{n}}^{n} U_{2}^{n} V_{1, n} V_{2, n} g\right\|$ when $n \rightarrow$ $\infty$ for $g \in \mathscr{A}_{\xi, \hat{\theta}}$. We have

$$
\begin{gathered}
V_{1, n} V_{2, n} U_{1}^{-n} U_{2}^{-n} \mathscr{L} U_{1}^{n} U_{2}^{n} V_{1, n} V_{2, n} g= \\
V_{1, n} V_{2, n} U_{1}^{-n} U_{2}^{-n} \mathscr{L} U_{1}^{n} U_{2}^{n}\left(V_{1, n} V_{2, n}-I\right) g+V_{1, n} V_{2, n} U_{1}^{-n} U_{2}^{-n} \mathscr{L} U_{1}^{n} U_{2}^{n} g .
\end{gathered}
$$

By (5.22) and the boundedness of $V_{j, n}, U_{j}^{ \pm n}$ and $\mathscr{L}$ we see that the first term in the right-hand side of (5.24) tends to zero strongly and uniformly for $g \in \mathscr{A}_{\xi, \hat{\theta}}$ when $n \rightarrow \infty$. The second term is equal to

$$
\begin{aligned}
& V_{1, n} V_{2, n} U_{1}^{-n} U_{2}^{-n}\left[\mathscr{L}, U_{1}^{n} U_{2}^{n}\right] g+V_{1, n} V_{2, n} \mathscr{L}_{g} \\
= & V_{1, n} V_{2, n} U_{1}^{-n} U_{2}^{-n}\left[\mathscr{L}, U_{1}^{n} U_{2}^{n}\right] g+\left(V_{1, n} V_{2, n}+I\right) \mathscr{L} g-\mathscr{L} g .
\end{aligned}
$$

Because $\mathscr{L}$ is a pseudodifferential operator of polynomial coefficients it follows from (5.22) that the second term of (5.25) tends to zero strongly and uniformly on $\mathscr{A}_{\xi, \hat{\theta}}$ when $n \rightarrow \infty$ for any $g \in \mathscr{A}_{\xi, \hat{\theta}}, \quad(g \neq 0)$.

Step 6. Let $g \in \mathscr{A}_{\xi, \hat{\theta}}, \quad(g \neq 0)$. We replace $g$ in (5.17) with $g /\|g\|$. By (5.19), (5.21) and the arguments in the above we have, if $n$ is sufficiently large

$$
\left\|\mathscr{L} \frac{g}{\|g\|}\right\|+\left\|V_{1, n} V_{2, n} U_{1}^{-n} U_{2}^{-n}\left[\mathscr{L}, U_{1}^{n} U_{2}^{n}\right] \frac{g}{\|g\|}\right\| \geq \frac{A}{2}
$$

Because $\mathscr{K}:=\left[\mathscr{L}, U_{1}^{n} U_{2}^{n}\right]$ is a pseudodifferential operator of order -1 we have (5.6).

Proof of the necessity of (2.6). Let $\xi \in \mathbb{Z}_{+}^{2}$ and $t \in \mathbb{N}$. We set

$$
g_{t}(\theta):=\phi(\theta) e^{\imath t \theta \xi}, \quad \phi(\theta)=\varphi\left(\frac{\theta-\widehat{\theta}}{\kappa}\right) .
$$

Clearly, $g_{t} \in \mathscr{A}_{\xi, \hat{\theta}}$ and the Fourier coefficient $\hat{g}_{t}$ of $g_{t}$ is given by $\hat{g}_{t}=\widehat{\varphi}((\eta-$ $t \xi) \kappa) \kappa^{2} e^{-i \hat{\theta}(\eta-t \xi)}$

By Lebesgue's dominated convergence theorem we have

$$
\lim _{t \rightarrow \infty} e^{-\imath t \theta \xi} \mathscr{L} g_{t}=\lim _{t \rightarrow \infty} \sum_{\eta \in \mathbb{Z}^{2}} L\left(e^{i \theta}, \frac{\eta}{|\eta|}\right) \kappa^{2} e^{-i \hat{\theta}(\eta-t \xi)} \widehat{\varphi}((\eta-t \xi) \kappa) e^{i \theta(\eta-t \xi)}
$$




$$
\begin{aligned}
& =\lim _{t \rightarrow \infty} \sum_{\zeta \in \mathbb{Z}^{2}-t \xi=\mathbf{Z}^{2}} L\left(e^{i \theta}, \frac{\zeta+t \xi}{|\zeta+t \xi|}\right) \kappa^{2} e^{-i \hat{\theta} \zeta} \widehat{\varphi}(\zeta \kappa) e^{i \theta \zeta} \\
& =L\left(e^{i \theta}, \frac{\xi}{|\xi|}\right) \sum_{\zeta \in Z^{2}} \kappa^{2} e^{-i \hat{\theta} \zeta} \bar{\varphi}(\zeta \kappa) e^{i \theta \zeta}=L\left(e^{i \theta}, \frac{\xi}{|\xi|}\right) \phi(\theta) .
\end{aligned}
$$

Again, by Lebesgue's dominated convergence theorem we have

$$
\lim _{t \rightarrow \infty}\left\|\mathscr{L} g_{t}\right\|=\left\|L\left(e^{i}, \frac{\xi}{|\xi|}\right) \phi\right\|
$$

In order to estimate $\left\|\mathscr{K} g_{t}\right\|$ with $\mathscr{K}$ given in (5.6) we recall that $\mathscr{K}$ is a pseudodifferential operator of order -1 . Therefore $\left\|K g_{t}\right\|$ is bounded by

$$
\begin{aligned}
\sum_{\eta}|\widehat{\varphi}((\eta-t \xi) \kappa)|^{2}\langle\eta\rangle^{-2} & =\sum_{\zeta \in \mathbf{Z}^{2}-t \xi}|\widehat{\varphi}(\zeta \kappa)|^{2}\langle\zeta+t \xi\rangle^{-2} \\
& =t^{-2} \sum_{\mid}|\widehat{\varphi}(\zeta \kappa)|^{2}\left\langle t^{-1} \zeta+\xi\right\rangle^{-2}
\end{aligned}
$$

The last term tends to zero when $t \rightarrow \infty$. On the other hand, by definition we have $\left\|g_{t}\right\|=\|\phi\|$.

By substituting (5.27) - (5.28) into (5.6) we obtain

$$
\|\phi\| \leq C\|L(\cdot, \xi /|\xi|) \phi\|
$$

Here we note that the constant $C$ in (5.29) is independent of $\phi$. It follows that

$$
\inf _{z \in T^{2}}|L(z, \xi /|\xi|)| \geq C^{-1}
$$

Indeed, if otherwise there exist $z_{\nu} \in \mathbb{T}^{2}$ and $\xi_{\nu} \rightarrow \infty$ such that

$$
\left|L\left(z_{\nu}, \xi_{\nu} /\left|\xi_{\nu}\right|\right)\right| \leq C_{0}<C^{-1}
$$

Passing to the subsequence, if necessary one may assume that $z_{\nu} \rightarrow z_{0}$ and $\xi_{\nu} /|\xi|$ $\rightarrow \xi_{0}(\nu \rightarrow \infty)$. Hence in a small neighborhood of $z_{0} \equiv e^{i \bar{\theta}}$ we have

$$
\mid L\left(z, \xi_{\nu} /\left|\xi_{\nu}\right|\right) \leq\left(C_{0}+C^{-1}\right) / 2
$$

If we take $\phi(\theta):=\varphi((\theta-\widehat{\theta}) / \kappa)$ with support contained in a small neighborhood of $\bar{\theta}$ by an appropriate choice of $\kappa$ we have 


$$
\left\|L\left(\cdot, \xi_{\nu} /\left|\xi_{\nu}\right|\right) \phi\right\|=\left(\int\left|L\left(e^{i \theta}, \xi_{\nu} /\left|\xi_{\nu}\right|\right) \phi(\theta)\right|^{2} d \theta\right)^{1 / 2} \leq \frac{1}{2}\left(C_{0}+C^{-1}\right)\|\phi\|
$$

This contradicts to (5.29). Hence we have proved (5.30). This proves (2.6). [5.

\section{\$6. Proof of Theorem 4.3. - the Necessity of $(2.7)$}

In this section we shall prove the necessity of (2.7) in Theorem 4.3. We use the same notations as in the previous sections unless otherwise is stated. We first prepare two lemmas. Let $\mathscr{L}\left(H_{1}^{2}\right)$ be the space of bounded linear operators on $H_{1}^{2}$ into $H_{1}^{2}$ and let $C_{\infty} \equiv C_{\infty}\left(H_{1}^{2}\right)$ be the space of linear compact operators on $H_{1}^{2}$. We denote the variable in $\mathbb{T}^{2}=\mathbb{R}^{2} / 2 \pi \mathbb{Z}^{2}$ by $\theta=\left(\theta_{1}, \theta_{2}\right)$. For a continuous function $\phi\left(\theta_{2}\right)$ on $\mathbb{T}^{2}$ depending only on $\theta_{2}$ we denote by $M(\phi)$ the multiplication operator by $\phi, M(\phi) u:=\phi u$ for $u \in L^{2}$. We denote by $I \otimes M$ $(\phi)$ the tensor product of $I$ and $M(\phi)$. For $0 \leq \gamma \leq 2 \pi$ we define a pseudodifferential operator $\mathscr{L}^{2, r}\left(\theta_{1}, D_{\theta}\right)$ by

$$
\mathscr{L}^{2, r}\left(\theta_{1}, D_{\theta}\right):=\mathscr{L}\left(\theta_{1}, \gamma, D_{\theta}\right) .
$$

Let $T_{1}$ be defined by (5.3) with $j=1$. Then we have

Lemma 6.1. For any $\varepsilon>0$ we can find a smooth function $\phi\left(\theta_{2}\right)$ satisfying 0 $\leq \phi \leq 1$ and $\phi \equiv 1$ in some neighborhood of $\theta_{2}=\gamma$ such that, for $\Psi=I \otimes M(\phi)$

$$
\left\|\left(T_{1}-\pi_{1} \mathscr{L}^{2, r}\left(\theta_{1}, D_{\theta}\right) \pi_{1}\right) \Psi\right\|<\varepsilon
$$

where the norm is understood in $L\left(H_{1}^{2}\right) / C_{\infty}\left(H_{1}^{2}\right)$.

Proof. We note that pseudodifferential operators of negative order define compact operators on $L^{2}\left(\mathbb{T}^{2}\right)$. Because $\mathscr{L}$ is a pseudodifferential operator with trigonometric polynomial coefficients the operator $\mathscr{L}$ can be written in the following form modulo compact operators

$$
\mathscr{L}\left(\theta, D_{\theta}\right)=\sum_{k \in \mathbb{Z}}^{\text {finite }} a_{k}\left(\theta_{2}, D_{\theta}\right) e^{\imath k \theta_{1}}
$$

for some pseudodifferential operators' $a_{k}\left(\theta_{2}, D_{2}\right)$. In view of $(6.1)$, a similar representation holds for $\mathscr{L}^{2, r}\left(\theta_{1}, D_{\theta}\right)$ modulo compact operators. Therefore, by the definition of $T_{1}$ we have 
(6.4)

$$
\begin{aligned}
\left(T_{1}-\pi_{1} \mathscr{L}^{2, \gamma}\left(\theta_{1}, D_{\theta}\right) \pi_{1}\right) \Psi & =\left(\pi_{1} \sum_{k} a_{k}\left(\theta_{2}, D_{\theta}\right) e^{\imath k \theta_{1}} \pi_{1}-\pi_{1} \sum_{k} a_{k}\left(\gamma, D_{\theta}\right) e^{\imath k \theta_{1}} \pi_{1}\right) \Psi \\
& =\sum_{k}\left(a_{k}\left(\theta_{2}, D_{\theta}\right)-a_{k}\left(\gamma, D_{\theta}\right)\right) \pi_{1} e^{\imath k \theta_{1}} \pi_{1}(I \otimes M(\phi)) .
\end{aligned}
$$

Because the summation in $k$ in (6.4) is a finite sum it is sufficient to show that each term in (6.4) tends to zero if supp $\phi$ tends to a point $\theta_{2}=\gamma$. Indeed, if we set $S_{k}=\pi_{1} e^{\imath k \theta_{1}} \pi_{1}$ and write $a_{k}\left(\theta_{2}, D_{\theta}\right)=\sum_{m} a_{k m}\left(D_{\theta}\right) e^{\imath m \theta_{2}}$ we have, for $u \in$ $L^{2}\left(\mathbf{T}^{2}\right)$

$$
\begin{aligned}
& \left(a_{k}\left(\theta_{2}, D_{\theta}\right)-a_{k}\left(\gamma, D_{\theta}\right)\right) S_{k}(I \otimes M(\phi)) u=\left(a_{k}\left(\theta_{2}, D_{\theta}\right)-a_{k}\left(\gamma, D_{\theta}\right)\right) \phi\left(\theta_{2}\right) S_{k} u . \\
= & \sum_{m} a_{k m}\left(D_{\theta}\right)\left(e^{i m \theta_{2}}-e^{i m \gamma}\right)\left(\phi S_{k} u\right)=\sum_{m} a_{k m}\left(D_{\theta}\right)\left(\left(e^{i m \theta_{2}}-e^{i m \gamma}\right)\left(\phi S_{k} u\right) .\right.
\end{aligned}
$$

Because the summation with respect to $m$ in (6.5) is a finite sum it is sufficient to estimate the term $a_{k m}\left(D_{\theta}\right)\left(\left(e^{\imath m \theta_{2}}-e^{\imath m r}\right) \phi S_{k} \imath \imath\right)$. Because $a_{k m}\left(D_{\theta}\right)$ is order zero and its $L^{2}$ norm is bounded, the $L^{2}$ norm of the term can be estimated by $A\left\|S_{k} u\right\|$. where $A \rightarrow 0$ if supp $\phi$ tends to a point $\theta_{2}=\gamma$. Therefore it follows that, modulo compact operators,

$$
\left\|a_{k m}\left(D_{\theta}\right)\left(\left(e^{\imath m \theta_{2}}-e^{\imath m r}\right) \phi S_{k} u\right)\right\| \leq C\left\|\left(e^{\imath m \theta_{2}}-e^{i m r \gamma}\right) \phi S_{k} u\right\| \leq C A\left\|S_{k} u\right\| \leq C A\|u\|,
$$

for some $C>0$, where we have used

$$
\left\|S_{k} u\right\|=\left\|\pi_{1} e^{\imath k \theta_{1}} \pi_{1} u\right\| \leq\left\|e^{\imath k \theta_{1}} \pi_{1} u\right\|=\left\|\pi_{1} u\right\| \leq\|u\| .
$$

This proves Lemma 6.1.

Let $\varphi\left(\theta_{1}\right) \in C^{\infty}(\mathbf{T}) \cap H^{2}(\mathbf{T})$ and $\phi\left(\theta_{2}\right) \in C^{\infty}(\mathbf{T})$. We define a function class $\mathscr{F}$ by

$$
\mathscr{F}:=\left\{\varphi\left(\theta_{1}\right) \phi\left(\theta_{2}\right) e^{\imath m \theta_{2}} ; m=1,2, \ldots\right\} \subset H_{1}^{2}
$$

Then we have

Lemma 6.2. Under the same assumptions as in Theorem 4.3 there exist compact operators $K$ and $\mathscr{K}^{*}$ on $H_{1}^{2}$ such that the following inequalities hold 


$$
\begin{gathered}
\left\|\pi_{1} \mathscr{L}_{1} g\right\|+\left\|\mathscr{K}_{g}\right\| \geq C\|g\|, \quad \forall g \in \mathscr{F}, \\
\left\|\pi_{1} \mathscr{L}^{*} \pi_{1} g\right\|+\left\|\mathscr{K}^{*} g\right\| \geq C\|g\|, \quad \forall g \in \mathscr{F},
\end{gathered}
$$

where $\mathscr{L}^{*}$ denotes the adjoint operator on $L^{2}$ of $\mathscr{L}$.

Proof. We shall prove (6.9). The inequality (6.10) will be proved similarly if we note Lemma 3.6. It follows from (5.10) that

$\left\|U_{1}^{-n} \pi_{1} \pi_{2} \mathscr{L}\left(\theta, D_{\theta}\right) \pi_{1} \pi_{2} U_{1}^{n} g\right\|+\left\|\pi_{1} \pi_{2} K \pi_{1} \pi_{2} U_{1}^{n} g\right\|+A\left\|U_{1}^{-n} Q_{2} U_{1}^{n} g\right\| \geq A\|g\| . \quad \forall g \in \mathscr{F}$.

If $g \neq 0$ we set $h:=g /\|g\|$ in (6.11). We obtain

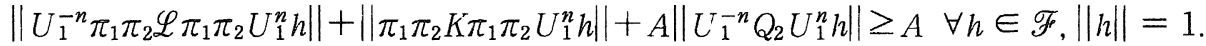

The third term in the left-hand side of (6.12) can be estimated by similar calculations as in (5.19). In fact, we have that, for any $\varepsilon>0$ there exists $n$ such that, for all $h \in \mathscr{F}$

$$
\left\|U_{1}^{-n} Q_{2} U_{1}^{n} h\right\|<\varepsilon .
$$

In order to estimate the first term we recall that $\left[U_{1}, \pi_{1}\right]=0$. Hence we have

$$
\begin{aligned}
U_{1}^{-n} \pi_{1} \pi_{2} \mathscr{L} \pi_{1} \pi_{2} U_{1}^{n} h & =V_{1, n} U_{1}^{-n} \pi_{1} \mathscr{L} \pi_{1} U_{1}^{n} V_{1, n} h=V_{1, n} \pi_{1} U_{1}^{-n} \mathscr{L} U_{1}^{n} \pi_{1} V_{1, n} h \\
& =V_{1, n} \pi_{1} \mathscr{L} \pi_{1} V_{1}^{n} h+V_{1, n} \pi_{1} U_{1}^{-n}\left[\mathscr{L}, U_{1}^{n}\right] \pi_{1} V_{1, n} h .
\end{aligned}
$$

The second term is a compact operator. As to the first term we have

$$
V_{1, n} \pi_{1} \mathscr{L}_{1} V_{1, n} h=\left(V_{1, n}-I\right) \pi_{1} \mathscr{L} \pi_{1} V_{1, n} h+\pi_{1} \mathscr{L} \pi_{1}\left(V_{1, n}-I\right) h+\pi_{1} \mathscr{L} \pi_{1} h .
$$

The first and the second term of the right-hand side of (6.15) tend to zero uniformly in $h \in \mathscr{F}$ by the definition of $V_{1, n}$ because $\mathscr{L}$ is of trigonometric polynomial coefficients. Therefore (6.15) implies that

$$
V_{1, n} \pi_{1} \mathscr{L} \pi_{1} V_{1, n} h \rightarrow \pi_{1} \mathscr{L} \pi_{1} h \text { uniformly for } h \in \mathscr{F}
$$

Hence it follows from (6.13) that, for sufficiently large $n$ 


$$
\left\|\pi_{1} \mathscr{L} \pi_{1} h\right\|+\|\mathscr{K} h\| \geq C, \quad \forall h \in \mathscr{F},\|h\|_{1}=1
$$

where $\mathscr{K}: H_{1}^{2} \rightarrow H_{1}^{2}$ is a compact operator. This proves $(6.9)$.

Proof of the necessity of (2.7). Let $a_{k}\left(\theta_{2}, \eta\right)$ be the symbol of $a_{k}\left(\theta_{2}, D_{\theta}\right)$ in (6.3). We define a function $A_{r}\left(\theta_{1}\right)$ by

$$
A_{\gamma}\left(\theta_{1}\right)=\sum_{k} a_{k}(\gamma, 0,1) e^{i k \theta_{1}}
$$

Namely we set $\theta_{2}=\gamma$ and $\eta=(0,1)$. We note that $A_{\gamma}\left(\theta_{1}\right)$ is a trigonometric polynomial of $\theta_{1}$. We then define a Toeplitz operator $\mathscr{T}\left(A_{\gamma}\right)$ with symbol $A_{\gamma} \equiv$ $A_{r}\left(\theta_{1}\right)$ by

$$
\mathscr{T}\left(A_{r}\right):=\pi_{1} A_{\gamma} ; H^{2}(\mathbb{T}) \rightarrow H^{2}(\mathbb{T})
$$

We want to show that $\mathscr{T}\left(A_{\gamma}\right)$ is invertible. By the condition (2.6) and the definition of $\mathscr{T}\left(A_{\gamma}\right)$ we know that $\mathscr{T}\left(A_{\gamma}\right)$ is a Fredholm operator. (cf. Theorem 2.42 of [3]). Hence, in order to prove (2.7) it is sufficient to show that

$$
\operatorname{Ker} \mathscr{T}\left(A_{\gamma}\right)=\operatorname{Ker} \mathscr{T}\left(A_{\gamma}\right)^{*}=\{0\}
$$

where $\mathscr{T}\left(A_{\gamma}\right)^{*}$ is the adjoint operator of $\mathscr{T}\left(A_{\gamma}\right)$.

Let $\varphi \equiv \varphi\left(\theta_{1}\right) \in H^{2}(\mathbb{T})$ satisfy that $\mathscr{T}\left(A_{\gamma}\right) \varphi=0$, that is $\pi_{1} A_{\gamma} \varphi=0$. We want to show that $\varphi\left(\theta_{1}\right)$ is smooth. Because $A_{r}$ is a trigonometric polynomial and $\varphi\left(\theta_{1}\right) \in H^{2}(\mathbb{T})$ the relation $\pi_{1} A_{r} \varphi=0$ implies that $A_{r} \varphi$ is a trigonometric polynomial with only negative Fourier coefficients. It follows that $g:=A_{r} \varphi$ $\in C^{\infty}(\mathbf{T})$. Because $A_{\gamma} \neq 0$ on $\mathbf{T}$ by (2.6) and the definition of $A_{r}$ we see that $\varphi=g / A_{r}$ is smooth.

For a smooth function $\phi\left(\theta_{2}\right)$ on $\mathbb{T}$ which will be determined later we set $\Psi$ $=I \otimes M(\phi)$. We consider the function class $\mathscr{F},(6.8)$ defined by these $\varphi$ and $\phi$. Then we have

$$
T_{1} \Psi=\mathscr{T}_{\gamma} \Psi+\left(T_{1}-\mathscr{T}_{\gamma}\right) \Psi
$$

where $T_{1}$ is given by (5.3) and we set $\mathscr{T}_{r}=\pi_{1} \mathscr{L}^{2, \gamma} \pi_{1}$ for the sake of simplicity. If we set $u=\phi\left(\theta_{1}\right) e^{\imath m \theta_{2}}(m=1,2, \ldots)$, we see that

$$
g:=\Psi u=\phi \phi e^{\imath m \theta_{2}} \in \mathscr{F} .
$$

It follows from (6.9) that 


$$
\left\|\mathscr{T}_{r} g^{\prime}\right\|+\left\|\left(T_{1}-\mathscr{T}_{\gamma}\right) g\right\|+\|\mathscr{K} g\| \geq C\|g\|, \quad g=\Psi u=\phi \phi e^{i m \theta_{2}}
$$

By Lemma 6.1 we can estimate the second term in the left-hand side of (6.20). In fact, for any $\varepsilon>0$ there exists a smooth function $\phi \neq 0$ on $\mathbb{T}$ such that $\Psi:=I \otimes M(\phi)$ satisfies, modulo compact operators

$$
\left\|\left(T_{1}-\mathscr{T}_{\gamma}\right) \Psi\right\|<\varepsilon
$$

We shall estimate the first term in (6.20). Let $\phi\left(\theta_{1}\right)=\sum_{j} \widehat{\phi}, e^{i j \theta_{1}}$ and $\phi\left(\theta_{2}\right)=\sum_{\ell} \phi_{\ell} e^{i \ell \theta_{2}}$ be Fourier expansions of $\phi$ and $\phi$, respectively. For $A$ $>0$ chosen later at (6.27) and a positive integer $m$ we set

$$
\phi_{m}\left(\theta_{1}\right)=\sum_{0 \leq j \leq m A} \widehat{\phi}_{\jmath} e^{i j \theta_{1}}, \quad \phi_{m}\left(\theta_{2}\right)=\sum_{|\ell| \leq m / 2} \widehat{\phi}_{\ell} e^{\ell \ell \theta_{2}}
$$

Then we have

$$
\begin{aligned}
\mathscr{T}_{r} g & =\mathscr{T}_{r} \phi \phi e^{i m \theta_{2}}=\mathscr{T}_{r}\left(\phi_{m}+\phi-\phi_{m}\right)\left(\phi_{m}+\phi-\phi_{m}\right) e^{i m \theta_{2}} \\
& =\mathscr{T}_{r}\left(\phi_{m} \phi_{m}+R_{m}\right) e^{i m \theta_{2}}=\mathscr{T}_{r} \phi_{m} \phi_{m} e^{i m \theta_{2}}+\mathscr{T}_{r} R_{m} e^{i m \theta_{2}}
\end{aligned}
$$

where

$$
R_{m}=\left(\phi-\phi_{m}\right) \phi_{m}+\phi_{m}\left(\phi-\phi_{m}\right)+\left(\phi-\phi_{m}\right)\left(\phi-\phi_{m}\right) .
$$

We shall estimate $\mathscr{T}_{\gamma} R_{m} e^{\imath m \theta_{2}}$. We first note that $\left\|R_{m}\right\|$ is rapidly decreasing when $m \rightarrow \infty$, namely, for $k=1,2, \ldots$

$$
\left\|R_{m}\right\| m^{k} \rightarrow 0 \quad(m \rightarrow \infty)
$$

Since $\mathscr{T}_{\gamma}$ is $L^{2}$ bounded it follows that

$$
\left\|\mathscr{T}_{r} R_{m} e^{i m \theta_{2}}\right\| \leq C\left\|R_{m} e^{i m \theta_{2}}\right\|=C\left\|R_{m}\right\|
$$

for some $\quad C>0$.

We shall estimate $\mathscr{T}_{r} \phi_{m} \psi_{m} e^{i m \theta_{2}}$. In what follows we denote constants independent of $m$ by $C$ for the sake of simplicity. Because $m \geq 0$ and $\phi \in H^{2}(\mathbb{T})$ we have

$$
\mathscr{T}_{r} \phi_{m}\left(\theta_{1}\right) \phi_{m}\left(\theta_{2}\right) e^{i m \theta_{2}}=\pi_{1} \sum_{k ; \text { finite }} a_{k}\left(\gamma, D_{\theta}\right) e^{i k \theta_{1}} \pi_{1} \phi_{m}\left(\theta_{1}\right) \phi_{m}\left(\theta_{2}\right) e^{i m \theta_{2}}
$$




$$
\begin{aligned}
& =\pi_{1} \sum_{k} a_{k}\left(\gamma, D_{\theta}\right) e^{i k \theta_{1}} \phi_{m}\left(\theta_{1}\right) \phi_{m}\left(\theta_{2}\right) e^{\imath m \theta_{2}} \\
& =\pi_{1} \sum_{0 \leq j \leq m A,|\ell| \leq m / 2, k} \widehat{\phi}_{j} \widehat{\phi}_{\ell} a_{k}\left(\gamma, D_{\theta}\right) e^{i(k+j) \theta_{1}+\imath(m+\ell) \theta_{2}} \\
& =\pi_{1} \sum_{k} a_{k}(\gamma, k+j, \ell+m) \widehat{\phi}_{j} \widehat{\phi}_{\ell} e^{\imath(k+j) \theta_{1}+i(m+\ell) \theta_{2}} .
\end{aligned}
$$

We note that $T\left(A_{\gamma}\right) \phi_{m}\left(\theta_{1}\right) \phi_{m}\left(\theta_{2}\right) e^{\imath m \theta_{2}}$ has a similar expression as (6.26) where $a_{k}(\gamma, k+j, \ell+m)$ is replaced by $a_{k}(\gamma, 0,1)$. By the homogenity of $a_{k}(\gamma, \eta)$ in $\eta$, for any $\varepsilon>0$ there exist an $m_{0}>0$ and a $A>0$ such that,

(6.27) $\left|a_{k}(\gamma, k+j, \ell+m)-a_{k}(\gamma, 0,1)\right|<\varepsilon \quad 0 \leq \forall j \leq m A, \forall|\ell| \leq m / 2, \forall m \geq m_{0}$.

It follows from $(6.17),(6.18),(6.26)$ and $(6.27)$ that, for $m \geq m_{0}$

$$
\begin{gathered}
\left\|\left(\mathscr{T}_{\gamma}-\mathcal{T}\left(A_{\gamma}\right)\right) \phi_{m} \psi_{m} e^{i m \theta_{2}}\right\|=\left\|\pi_{1} \sum_{k}\left(a_{k}\left(\gamma, D_{\theta}\right)-a_{k}(\gamma, 0,1)\right) e^{i k \theta_{1}} \phi_{m} \psi_{m} e^{i m \theta_{2}}\right\| \\
\leq \sum_{k}\left\|\left(a_{k}\left(\gamma, D_{\theta}\right)-a_{k}(\gamma, 0,1)\right) e^{i k \theta_{1}} \phi_{m} \psi_{m} e^{i m \theta_{2}}\right\| \leq C \varepsilon\|\phi\|\|\phi\|
\end{gathered}
$$

for some $C>0$. On the other hand, since $\mathscr{T}\left(A_{\gamma}\right) \phi=0$ it follows that

$$
\begin{gathered}
\left\|\mathscr{T}\left(A_{r}\right) \phi_{m} \psi_{m} e^{2 m \theta_{2}}\right\|=\left\|\phi_{m} e^{\imath m \theta_{2}} \mathscr{T}\left(A_{r}\right) \phi_{m}\right\| \\
=\left\|\phi_{m} e^{i m \theta_{2}} \mathscr{T}\left(A_{r}\right)\left(\phi_{m}-\phi\right)\right\| \leq C\|\phi\|\left\|\phi_{m}-\phi\right\| \leq C \varepsilon\|\phi\|,
\end{gathered}
$$

for some $C>0$ if $m$ is sufficiently large. Therefore it follows from (6.28) and (6.29) that

$$
\begin{aligned}
\left\|\mathscr{T}_{r} \phi_{m} \psi_{m} e^{i m \theta_{2}}\right\| & \leq\left\|\left(\mathscr{T}_{r}-\mathscr{T}\left(A_{\gamma}\right)\right) \phi_{m} \psi_{m} e^{\imath m \theta_{2}}\right\|+\left\|\mathscr{T}\left(A_{\gamma}\right) \phi_{m} \phi_{m} e^{\imath m \theta_{2}}\right\| \\
& \leq C \varepsilon\|\phi\|\|\phi\|+C \varepsilon\|\phi\| .
\end{aligned}
$$

Hence it follows from $(6.23),(6.25)$ and $(6.30)$ that

(6.31) $\quad\left\|\mathscr{T}_{r} g\right\| \leq\left\|\mathscr{T}_{r} R_{m} e^{\imath m \theta_{2}}\right\|+\left\|\mathscr{T}_{r} \phi_{m} \phi_{m} e^{\imath m \theta_{2}}\right\| \leq C m^{-1}+C \varepsilon\|\phi\|\|\phi\|+C \varepsilon\|\phi\|$. 
By (6.21) there exists a compact operator $\widetilde{\mathcal{K}}$ such that for $g=\Psi_{u}=\phi \psi e^{\imath m \theta_{2}}, u$ $=\phi\left(\theta_{1}\right) e^{i m \theta_{2}}$

$$
\left\|\left(T_{1}-\mathscr{T}_{r}\right) g\right\| \leq \varepsilon\|u\|+\|\widetilde{\mathscr{K}} u\|=\varepsilon\|\phi\|+\left\|\widetilde{\mathscr{K}} \phi\left(\theta_{1}\right) e^{\imath m \theta_{2}}\right\| .
$$

Therefore, by $(6.20),(6.31)$ and the relations $g=\Psi u=\phi \psi e^{\imath m \theta_{2}}, u=\phi\left(\theta_{1}\right)$ $e^{i m \theta_{2}}$ we have

$$
C\|g\| \leq\|\mathscr{K} g\|+\varepsilon\|\phi\|+\left\|\widetilde{\mathscr{K}} \phi\left(\theta_{1}\right) e^{\imath m \theta_{2}}\right\|+C m^{-1}+C \varepsilon\|\phi\|\|\phi\|+C \varepsilon\|\phi\| .
$$

Since $g$ and $\phi\left(\theta_{1}\right) e^{\imath m \theta_{2}}$ weakly converge to zero when $m \rightarrow \infty$ and $\mathscr{K}, \widetilde{\mathcal{K}}$ are compact, it follows that $\mathscr{K} g$ and $\widetilde{K} \phi\left(\theta_{1}\right) e^{\imath m \theta_{2}}$ strongly converge to zero when $m$ $\rightarrow \infty$. Noting that $\|g\|=\|\phi\|\|\phi\|$ and letting $m \rightarrow \infty$ in (6.32) we obtain

$$
C\|\phi\|\|\phi\| \leq \varepsilon\|\phi\|+\varepsilon\|\phi\|\|\phi\|+C \varepsilon\|\phi\| .
$$

Because $\varepsilon>0$ is arbitrary we have $\|\phi\|\|\phi\|=0$. Since $\|\phi\| \neq 0$ we have $\phi$ $=0$. This proves that the kernel of $\mathscr{T}\left(A_{\gamma}\right)$ is trivial. If we make the same argument to the adjoint operator $\mathscr{T}\left(A_{\gamma}\right)^{*}$ we can show that Cokernel $\mathscr{T}\left(A_{r}\right)=$ $\{0\}$. This proves that $\mathscr{T}\left(A_{\gamma}\right)$ is invertible. By the well-known result of Toeplitz operators (cf. Theorem 2.42 of [3]) we have ind $A_{r}=0$. By the definition of $A_{r}$ this proves that ind ${ }_{1} L=0$. By changing the parts of $\theta_{1}$ and $\theta_{2}$ we can prove that $\operatorname{ind}_{2} L=0$. Hence we have proved (2.7). The proof of Theorem 4.3 is complete. 屁

\section{References}

[1] Bengel, G. and Gérard, R., Formal and convergent solutions of singular partial differential equations, Manuscripta Math., 38 (1982), 343-373.

[2] Boutet de Monvel. L. and Guillemin V.. The spectral theory of Toeplitz operators, Annals of Mathematics Studies, 99, Princeton University Press, 1981.

[3] Bottcher, A. and Silbermann, B., Analysis of Toeplitz operators, Springer Verlag, Berlin, 1990.

[4] Kashiwara, M., Kawai, T. and Sjostrand J., On a class of linear partial differential equations whose formal solutions always converge, Ark. fur Math. 17 (1979), 83-91.

[5] Komatsu, H., Linear ordinary differential equations with Gevrey coefficients. J. Differential Eqs., 45 (1982), 272-306.

[6] Malgrange, B., Sur les points singuliers des équations différentielles linéaires, Enseign. Math., 20 (1970), 146-176.

[7] Miyake. M. and Yoshino, M., Wiener-Hopf equation and Fredholm property of the Goursat problem in Gevrey space, Nagova Math. J.; 135 (1994), 165-196.

[8] - Fredholm property for differential operators on formal Gevrey space and Toeplitz operator method, Comptes rendus de l'Académie bulgare des Sciences, 47 (1994), 21-26.

[9] - Riemann-Hilbert factorization and Fredholm property of differential operators of 
irregular singular type, Ark. fur Math. 33 (1995), 323-341.

[10] Ramis, J.P., Théorèmes d'ındıces Gevrey pour les équations différentielles ordinaires, Mem. Amer. Math. Soc., 48 (1984). 
